\title{
Development of Transdermal Ondansetron Hydrochloride for the Treatment of Chemotherapy-Induced Nausea and Vomiting
}

\author{
Rajan Rajabalaya*, Ding Siok Chen and Sheba Rani Nakka David \\ Department of Pharmaceutical Technology, International Medical University, No. 126, Jalan Jalil Perkasa 19, Bukit Jalil 57000 , \\ Kuala Lumpur, Malaysia. \\ *For correspondence: Email: rajan_rajabalaya@imu.edu.my; Tel: +60 327317210; Fax: +60 386567229 \\ Received: 6 July 2012 \\ Revised accepted: 17 April 2013
}

\begin{abstract}
Purpose: To to develop and evaluate matrix-type ondansetron hydrochloride (OS) transdermal patch for the treatment of chemotherapy-induced nausea and vomiting.

Methods: Transdermal patches were prepared by solvent casting method using ethyl cellulose and polyvinyl pyrrolidone as matrix materials, and dibutyl phthalate and dibutyl sebacate as plasticizers. The formulations were evaluated for patch thickness, tensile strength, moisture content, water absorption capacity and drug content. In vitro drug release and permeation of the patches were determined using a Franz diffusion cell.

Results: the tensile strength of all the formulations was in the range from 6.09 to 9.85 Mpa indicating that the [patches were strong. Maximum drug release in $8 \mathrm{~h}$ for dibutyl phthalate DBP and dibutyl sebacate DBS patches was 38.9 (DB6) and $53.4 \%$ (DS3), respectively, which are significantly ( $p<$ 0.01) higher than the lowest values of 17.8 (for DB1) and $35.0 \%$ for (DS5), respectively. Drug release rate was 1.89 and $3.93 \mu \mathrm{g} / \mathrm{h} / \mathrm{cm}^{2}$, respectively with DS2 and DB2 showing the highest permeation rate of $5.39 \mathrm{\mu g} / \mathrm{h} / \mathrm{cm}^{2}$. Patches containing DBP followed Higuchi release model while patches formulated with DBS followed first order release kinetics.

Conclusion: Ondansetron matrix-type transdermal patches formulated with suitable amounts of chemical enhancers for better patient compliance are feasible.
\end{abstract}

Keywords: Ondansetron hydrochloride, Chemical enhancers, Plasticizers, Dibutyl phthalate, Dibutyl sebacate, Permeation, Patch.

Tropical Journal of Pharmaceutical Research is indexed by Science Citation Index (SciSearch), Scopus, International Pharmaceutical Abstract, Chemical Abstracts, Embase, Index Copernicus, EBSCO, African Index Medicus, JournalSeek, Journal Citation Reports/Science Edition, Directory of Open Access Journals (DOAJ), African Journal Online, Bioline International, Open-J-Gate and Pharmacy Abstracts

\section{INTRODUCTION}

Chemotherapy-induced nausea vomiting (CINV) is one of the most distressing side effects of chemotherapy; approximately $70 \%$ of patients who receive chemotherapy will experience some level of CINV [1]. CINV can cause significant discomfort and anxiety, dehydration, electrolyte imbalances, affect normal physical and mental function, and decrease quality of life [2]. Hence, some patients may choose to give up the beneficial chemotherapy in the end due to its side effects [1]. Although researchers ar keep finding more effective therapy for CINV every year, CINV still remains as an important issue [35]. None of the therapies has completely solved the problem; in fact, up to $30 \%$ of the patients xperience refractory nausea vomiting and also delayed CINV, even with the best antiemetic treatment [6].

Ondansetron (OS), a member of the serotonin 
(5-hydroxytryptamine) subtype 3 (5-HT3) group of receptor antagonists, can effectively act on chemoreceptor trigger zone of postrema to control nausea and vomiting. As OS does not inhibit dopamine subtype-2 receptors, it does not cause side effects such as extrapyramidal reactions [7]. Although OS is a potent antiemetic, including for patients receiving highly emetogenic agents, oral administration is only effective in patients who can swallow medications after chemotherapy [8].

Several disadvantages with oral, intravenous and rectal administration include extensive liver metabolism, low bioavailability, vomiting before drug absorption, rapid onset that may result in undesirable side effects, high clearance, short half-life, and low patient compliance [9]. To overcome these, Gwak et. al [8] had studied the feasibility of transdermal OS patch delivery using pressure sensitive adhesives. Other studies have shown that placing transdermal patch on a patient's skin for 24 - $48 \mathrm{~h}$ before chemotherapy is just as effective as the oral capsule [1]. Therefore, the objective of the present study was to optimize OS transdermal patch formulation for the treatment of CINV.

\section{EXPERIMENTAL}

\section{Materials}

Ondansetron hydrochloride was obtained as a gift from Aurobindo Chemicals, India. Ethyl cellulose (EC; ethoxy content 48.0 - $49.5 \%$, viscosity 18 to $22 \mathrm{mPa}$ ) was received from Dow Chemicals, Germany. Polyvinyl pyrrolidone (PVP K-30) was obtained as a gift from BASF Chemical Company, Germany. Dibutyl phthalate (DBP) and dibutyl sebacate (DBS) were purchased from Sigma-Aldrich Chemie $\mathrm{GmbH}$, Riedstr, and Merck Chemicals, Germany respectively. Linseed oil was received as a gift from John L Seatons \& Co Ltd, UK, and complied with British Pharmacopoeia (BP)/European harmacopoeia(EP) standard. L-menthol was purchased from Spectrum Chemical Mfg Corp, USA. All the solvents used were of analytical reagent grade.

\section{Preparation of the patches}

The polymers were accurately weighed and dissolved in $5 \mathrm{~mL}$ of chloroform.. The required amount of plasticizer, dibutyl phthalate (DBP) or dibutyl sebacate (DBS), was added next, followed by the addition of OS, and then gently stirred for 20 mins 15 - 20 min. For patch containing enhancers, the enhancers were added after the drug and stirred for another 15 -
$20 \mathrm{~min}$. The resulting homogenous solution was slowly poured within a stainless steel ring with a backing layer of aluminium foil and dried at room temperature for 48 h 24 - 48 h [11].

\section{Patch thickness}

Patch thickness was measured using a digital micrometer (Mitutoyo, Japan) [11]. The results are reported as amean of six readings (Table 1 ).

\section{Tensile strength}

Tensile strength ( $\mathrm{T}$ ) was measured using a tensilometer (Instron, UK), and calculated as in Eq 1.

$T=L_{\max } / A_{i}$

where $L_{\max }=$ maximum load and $A i=$ initial cross sectional area of the sample [11]. The results are reported as mean of three readings (Table 1).

\section{Drug content}

A circular patch $\left(1 \mathrm{~cm}^{2}\right)$ was cut, weighed and dissolved in chloroform. Chloroform Distilled water was added to dilute to $100 \mathrm{ml}$, the same solution filtered through whatman filter paper

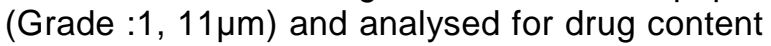
at $249 \mathrm{~nm}$ using a UV spectrophotometer (Perkin-Elmer). The same procedure was carried out for control (drug-free) film [11]. The results are reported as mean of three readings (Table 2).

\section{Moisture content}

The test patches were weighed individually and placed in a desiccator containing fused calcium chloride at $40{ }^{\circ} \mathrm{C}$ for $24 \mathrm{~h}$. The patches were reweighed until a constant weight was obtained. The moisture content was calculated as as percent loss in weight [11] and expressed as mean $(n=3$, Table 2$)$.

\section{Water absorption studies}

The patches were weighed individually, kept at room temperature for $24 \mathrm{~h}$ and then exposed to two relative humidities: $75 \%$ (saturated aqueous solution of sodium chloride) and $93 \%$ (saturated aqueous solution of ammonium hydrogen phosphate) in separate desiccators. The patches were re-weighed until constant weight was obtained. Water absorption capacity was calculated as percent gain in weight [11] and expressed as mean $(n=3$, Table 2$)$. 


\section{In vitro release studies}

In vitro release studies were carried out using Franz diffusion cell (Perme Gear, USA), whereby a piece of the circular patches was mounted over the donor compartment. The backing membrane side of the patch was exposed to the atmosphere while the receptor compartment was filled with freshly prepared phosphate buffered saline $(\mathrm{pH}$ 7.4). Temperature was maintained at $32{ }^{\circ} \mathrm{C}$ by circulating water through the water jacket and stirring at $5040-50 \mathrm{rpm}$. The patch was in contact with the receptor liquid surface. Samples $(0.5 \mathrm{~mL})$ were withdrawn at $1 \mathrm{~h}$ interval for $8 \mathrm{~h}$ and immediately replaced with the same volume of medium. Each sample was filtered, diluted suitably and analyzed spectrophotometrically at $249 \mathrm{~nm}$ [11]. The mean of three readings was taken. In order to investigate drug release mechanism, the data were fitted to zero order, first order, Higuchi and Korsmeyer-Peppas release models as in Eqs 1 - 4, respectively..

$$
M_{t}=M_{0}+K_{0}
$$

where $M_{t}$ is the amount of drug released at time $t, K_{0}$ is the apparent dissolution rate constant or zero-order release constant and $M_{0}$ is the initial amount of the drug in the solution as a result of 'burst' effect. In this model, the drug releases at constant rate.

$$
\ln M_{t}=\ln M_{0}+K_{1} t
$$

where $K_{1}$ is the first-order release constant. In this model, the drug released at any time, $\mathrm{t}$, is proportional to the residual drug inside the dosage form.

$$
M_{t}=M_{0}+K_{H} t^{(1 / 2)}
$$

where $K_{H}$ is the Higuchi release constant. This is the most widely used model to describe drug release from pharmaceutical matrices.

$$
M_{t} / M_{\infty}=\left(\begin{array}{ll}
M_{0} / M_{\infty}
\end{array}\right)+K_{K P} t^{n} \ldots \ldots . .(4)
$$

where $K_{K P}$ is a constant incorporating structural and geometric characteristic of the drug-dosage form and $n$ is the release exponent, indicative of the drug-release mechanism. Peppas used this $n$-value in order to characterize different release mechanisms. When $n=0.5$ (for a slab), the drug diffuses through and is released from the matrix with a quasi-fickian diffusion mechanism. For $n>0.5$, an anomalous, non-fickian drug diffusion occurs. When $n=1.0$, a non-fickian, Case II or zero-order release kinetics could be observed. For determining of the exponent $n$, the portion of the release curve where $M_{t} / M_{\infty}<0.6$ is only used. To use this equation it is also necessary that release occurs in one-dimensional way and that the system width/thickness or length/thickness relation be at least 10 , such as in transdermal delivery system.

\section{In vitro permeation studies}

A section of freshly excised albino mouse abdominal skin was shaved and removed skin fatty tissue with help of scrapper. The skin sample immersed in isotonic solution $10.9 \mathrm{~g}$ sodium chloride dissolved in $100 \mathrm{~mL}$ of reverse osmosis R.O water) for the purpose of remove the unwanted protiens. The test patch was bound intimately with help of adhesive on mouse skin and placed over the top of the donor compartment of Franz diffusion cell. The dermal side of the skin just touched the receptor liquid surface. All other analysis conditions were similar to those for in vitro release studies [11]. The mean of three readings was taken..

After reviewing all the in vitro factors affecting the mechanical, release and physiochemical properties of the patches, some of patches were selected for skin permeation studies.

\section{Statistical analysis}

Release data were evaluated with one-way analysis of variance (ANOVA). Where there was statistically significant difference, post-hoc Tukey-HSD (Honestly Significant Difference) test was performed. Differences between release data were considered statistically significant at $p$ $\leq 0.05$.

\section{RESULTS}

\section{Thickness and tensile strength}

The patch thickness and tensile strength results are shown in Table 1. Mean thicknesses varied from 0.195 to $0.235 \mathrm{~mm}$ while mean tensile strength was in the range 6.09 to $9.85 \mathrm{MPa}$. Thicknesses is gradually decreasing with increasing the amount of plasticizers and PVP percentages in the patch whereas tensile strengths increased which is most preferable physiochemical properties for the patient.

\section{Drug content, moisture content and water absorption of patches}

The results of assessment of drug content, moisture content and water absorption of patches are shown in Table 2 Drug content of patches ranged from 425.6 to $427.0 \mathrm{mcg} / \mathrm{cm}^{2}$, indicating a mean drug content of $>99 \%$ of the theoretical amount. 
Table 1: Transdermal ondansetron patch characteristics

\begin{tabular}{|c|c|c|c|c|c|}
\hline $\begin{array}{c}\text { Patch code } \\
\text { EC : OS } \\
350: 16(\mathrm{mg})\end{array}$ & $\begin{array}{l}\text { PVP } \\
\text { (mg) }\end{array}$ & \multicolumn{2}{|c|}{$\begin{array}{c}\text { Plasticizer } \\
(\%)\end{array}$} & Thickness (mm) & $\begin{array}{c}\text { Tensile strength } \\
\text { (MPa) }\end{array}$ \\
\hline DB 1 & 150 & \multirow{10}{*}{ DBP } & 30 & 0.217 & $6.54 \pm 0.37$ \\
\hline DB 2 & 150 & & 40 & 0.220 & $6.23 \pm 0.75$ \\
\hline DB 3 & 150 & & 50 & 0.223 & $6.09 \pm 0.35$ \\
\hline DB 4 & 200 & & 30 & 0.219 & $7.44 \pm 0.98$ \\
\hline DB 5 & 200 & & 40 & 0.223 & $7.24 \pm 0.23$ \\
\hline DB 6 & 200 & & 50 & 0.227 & $6.31 \pm 0.76$ \\
\hline DB 7 & 250 & & 10 & 0.221 & $8.86 \pm 0.39$ \\
\hline DB 8 & 250 & & 20 & 0.225 & $8.63 \pm 0.61$ \\
\hline DB 9 & 250 & & 30 & 0.228 & $8.18 \pm 0.16$ \\
\hline DB 10 & 250 & & 40 & 0.231 & $7.82 \pm 0.82$ \\
\hline DB 11 & 250 & \multirow{7}{*}{ DBS } & 50 & 0.235 & $7.59 \pm 0.94$ \\
\hline DS 1 & 150 & & 30 & 0.195 & $7.46 \pm 1.10$ \\
\hline DS 2 & 150 & & 40 & 0.199 & $7.31 \pm 0.53$ \\
\hline DS 3 & 150 & & 50 & 0.205 & $7.23 \pm 0.49$ \\
\hline DS 4 & 200 & & 30 & 0.198 & $9.85 \pm 1.07$ \\
\hline DS 5 & 200 & & 40 & 0.203 & $9.65 \pm 0.34$ \\
\hline DS 6 & 200 & & 50 & 0.208 & $8.23 \pm 0.51$ \\
\hline
\end{tabular}

Key: $E C=$ ethyl cellulose; $O \bar{S}=$ ondansetron hydrochloride; $P V P=$ polyvinyl pyrrolidone; $\overline{D B P}=$ dibutyl phthalate; $D B S=$ dibutyl sebacate

Table 2: Drug content, moisture content and water absorption of ondansetron patch

\begin{tabular}{ccccc}
\hline Patch code & $\begin{array}{c}\text { Drug content } \\
\left(\mathbf{m c g} / \mathbf{c m}^{2}\right)\end{array}$ & $\begin{array}{c}\text { Moisture content } \\
(\mathbf{w t} \text { \%) }\end{array}$ & \multicolumn{2}{c}{ Water absorption (wt \%) } \\
\cline { 3 - 5 } & 425.87 & 1.67 & $\mathbf{7 5 \%} \boldsymbol{R H}$ & $\mathbf{9 3 \%} \mathbf{R H}$ \\
\hline DB 1 & 426.11 & 1.42 & 2.10 & 2.38 \\
DB 2 & 426.39 & 1.24 & 1.90 & 2.19 \\
DB 3 & 426.52 & 1.97 & 1.76 & 2.08 \\
DB 4 & 426.92 & 1.86 & 2.38 & 2.81 \\
DB 5 & 426.78 & 1.73 & 2.16 & 2.68 \\
DB 6 & 425.98 & 2.73 & 2.04 & 2.47 \\
DB 7 & 426.85 & 2.67 & 2.86 & 3.18 \\
DB 8 & 426.09 & 2.56 & 2.67 & 3.02 \\
DB 9 & 426.83 & 2.17 & 2.49 & 2.81 \\
DB 10 & 426.91 & 2.03 & 2.27 & 2.69 \\
DB 11 & 425.58 & 1.16 & 2.18 & 2.49 \\
DS 1 & 425.99 & 1.02 & 1.37 & 2.07 \\
DS 2 & 426.31 & 0.86 & 1.18 & 1.81 \\
DS 3 & 426.14 & 1.42 & 1.06 & 1.57 \\
DS 4 & 426.57 & 1.28 & 1.97 & 2.19 \\
DS 5 & 426.83 & 1.15 & 1.81 & 2.09 \\
DS 6 & & & 1.56 & 1.82 \\
\hline
\end{tabular}

\section{In vitro drug release}

The release data over $8 \mathrm{~h}$ are shown in the Figures 1 and 2.. Higher amount of PVP in the patches have a greater dissolution of more and more PVP in the diffusion medium forms pores which increase the rate of diffusion. And also the percentage drug release is based on various factors like solubility of the drug in the polymer and solubility of drug in other ingredients. The hydrophobic plasticizers DBP and DBS and hydrophilic nature PVP copolymer give ideals release pattern. Furthermore, in terms of physical appearance, DBP patches were appeared to have better quality and smoothness than DBS patches $[11,15]$. 


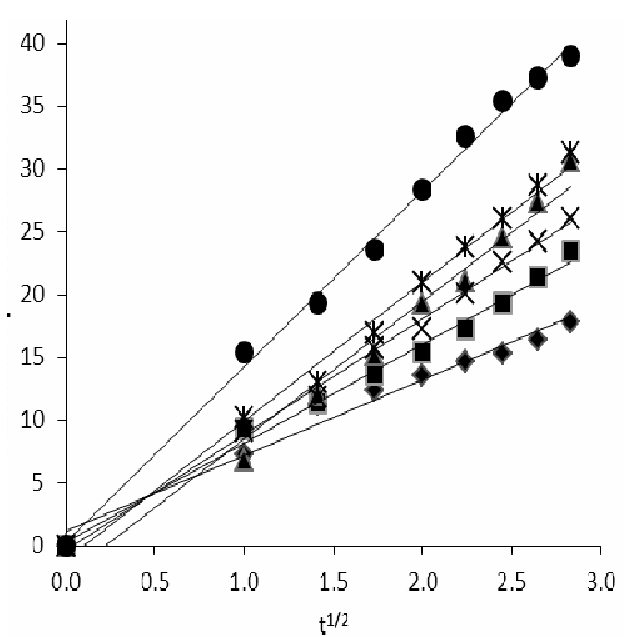

Figure 1: Release profile of patches containing DBP. Key: Formulations DB1 (»), DB2 (घ), DB3 $(\mathbf{\Lambda})$, DB4 $(\mathrm{x})$, DB5 $(*)$, DB6 $(\bullet)$

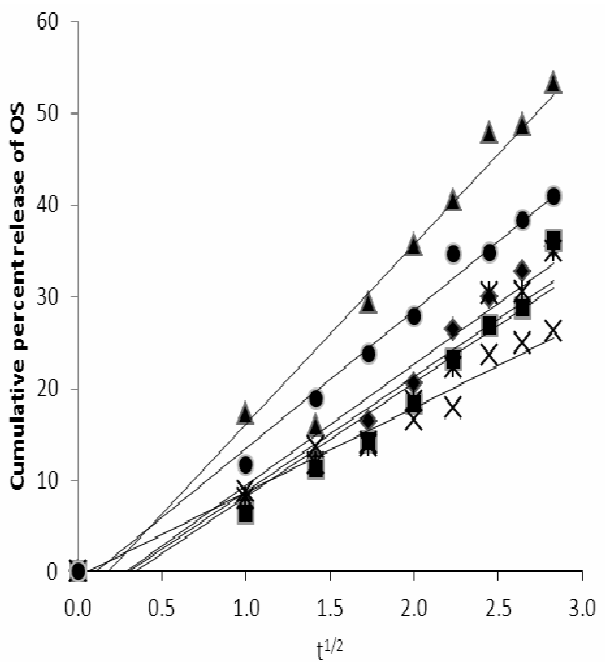

Figure 2: Release profiles of patches containing DBS. Key: Formulations DBS1 (•), DS2 (๘), DS3 $(\mathbf{\Delta})$, DS4 $(\mathrm{x})$, DS5 $(*)$, DS6 $(\bullet)$

Table 3: Release and kinetic data

\begin{tabular}{ccccccccc}
\hline \multirow{2}{*}{$\begin{array}{c}\text { Patch } \\
\text { code }\end{array}$} & \multicolumn{2}{c}{ Zero order } & \multicolumn{2}{c}{ First order } & \multicolumn{2}{c}{ Higuchi release } & \multicolumn{2}{c}{ Korsmeyer-peppas } \\
\cline { 2 - 8 } & $\mathrm{K}_{0}$ & $\mathrm{R}^{2}$ & $\mathrm{~K}_{1}$ & $\mathrm{R}^{2}$ & $\mathrm{~K}_{0.5}$ & $\mathrm{R}^{2}$ & $\mathrm{n}$ & $\mathrm{R}^{2}$ \\
\hline DB1 & 1.43 & 0.9876 & -0.097 & 0.9213 & 7.46 & 0.9966 & 0.61 & 0.9947 \\
DB2 & 1.86 & 0.9823 & -0.101 & 0.9139 & 9.68 & 0.9961 & 0.64 & 0.9951 \\
DB3 & 2.43 & 0.9839 & -0.088 & 0.8606 & 12.92 & 0.9977 & 0.57 & 0.9808 \\
DB4 & 2.30 & 0.9437 & -0.122 & 0.9679 & 10.36 & 0.9928 & 0.74 & 0.9653 \\
DB5 & 1.24 & 0.9838 & -0.081 & 0.9481 & 6.44 & 0.9975 & 0.49 & 0.9833 \\
DB6 & 1.46 & 0.9932 & -0.101 & 0.8831 & 7.68 & 0.9901 & 0.65 & 0.9988 \\
DS1 & 1.85 & 0.9721 & -0.076 & 0.9988 & 9.78 & 0.9982 & 0.48 & 0.9962 \\
DS2 & 1.60 & 0.9681 & -0.085 & 0.9969 & 8.30 & 0.9749 & 0.52 & 0.9746 \\
DS3 & 1.99 & 0.9588 & -0.111 & 0.9891 & 10.31 & 0.9728 & 0.68 & 0.9969 \\
DS4 & 1.52 & 0.9721 & -0.078 & 0.9959 & 8.01 & 0.9882 & 0.50 & 0.9969 \\
DS5 & 1.52 & 0.9689 & -0.081 & 0.9915 & 7.81 & 0.9793 & 0.49 & 0.9732 \\
DS6 & 1.67 & 0.9648 & -0.092 & 0.9832 & 8.63 & 0.9663 & 0.57 & 0.9816 \\
\hline
\end{tabular}

\section{In vivo permeation}

Cumulative drug permeation data through the best three patches are shown in Figure 3.

The percent release for DB2 at 3\% linseed oil was observed to be 1.5 times greater with respect to the same formulation without enhancer $(p \leq 0.05)$, whereas DB1 and DB3 at $3 \%$ linseed oil appeared to increase transport of OS, but this increased did not show a significant difference $(p>0.05)$. In general, a moderate increase in release of drug was achieved by incorporating 4\% linseed oil (DB 1-3) however; a definite increase in release of drug was only seen following the addition of $40 \%$ DBP and $4 \%$ linseed oil. Hence, the maximum percent release was seen at patch DB2 with $4 \%$ linseed oil, approximately 2.5 times greater to DB2 alone $(p \leq 0.05)$.

The increasing drug permeation rate with the increase in PVP content may be due to its antinucleating effect as well as different types of chemical enhancers affecting the permeation pattern.

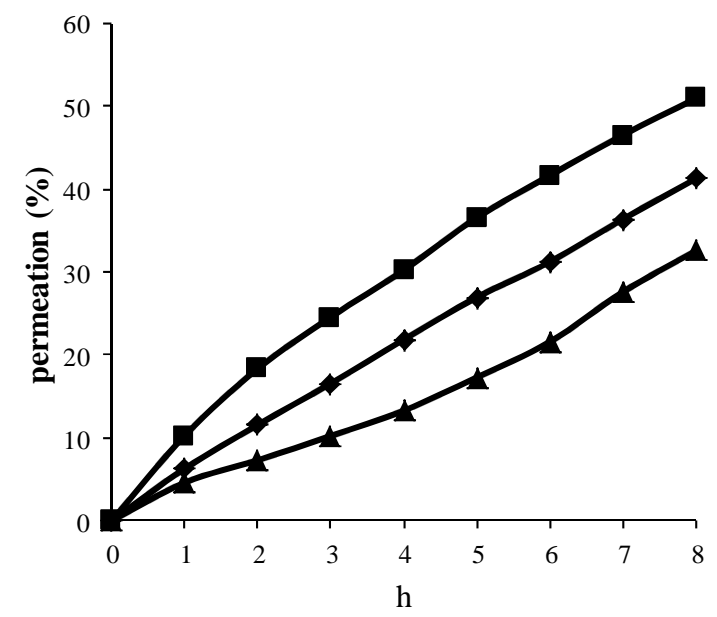

Figure 3: Permeation profile of patches containing DBP and $4 \%$ linseed oil

Key: DB1 (४), DB2 (-) and DB3 (4) 


\section{DISCUSSION}

The decrease in patch strength by the plasticizers is due to the interposition of the latter between polymer chains of the matrix, thus reduceing intermolecular forces and thereby, the mechanical strength of the patches [12]. Increase in patch strength by PVP may be attributed to the formation of crosslinks within the EC matrix, thus strengthening intermolecular bonds [13]. Generally, both plasticizers DBP and DBS did not show much difference on mechanical properties, though DBP containing patches showing slightly lower tensile strength owing to smaller size for incorporation than DBS. Less than $30 \%$ of DBS is required to produce a stiff, strong EC membrane which on iys own is brittle [14] Findings from our studies confirm this same observation.

Tensile strength is important to ascertain the maximum force that a patch can withstand while it is being stretched as well as indicate how well a patch can remain intact during biomechanical movement of the human body. For optimum elasticity on human usage, it has been that tensile strength should be $>4 \mathrm{MPa}$ [11].

Moisture content and water absorption capacity were largely dependent on the amounts of PVP and plasticizer in the patch. PVP, being hygroscopic, would readily absorb moisture from the atmosphere into the patch, which explains the higher moisture content and water absorption of patches the higher the PVP level [11]. Since both DBP and DBS are hydrophobic, increasing the content of plasticizer reduced moisture content and water uptake of the patch.

The percentage of release increased when percentage of plasticizer was also increased (DB3 > DB2 > DB1, etc). The reverse, however, was seen for PVP incorporation (DB4 > DB1; DB5 > DB2; DB6 > DB3) as a rise in the polymer content enhanced release rate as a result of invreased water absorption which caused swelling of the polymeric matrix, thus widening the diffusion pathways and facilitating drug diffusion.

DBS is suitable for rapid release while DBP is ideal for prolong release [17]. This explains why patches containing DBS showed higher and faster release than those containing DBP. The reason may be due to breakdown of secondary polymer chain bonding by DBS thus providing more space for the drug to diffuse.

For some of the formulations, the presence of linseed oil decreased OS release from the patches without enhancer. Low release rate may be due to the higher hydrophobicity of the patches as well as formation of drug-in-oil dispersion which would retard the diffusion of drug from the patches [24].

From the figure and table 3 shows that the drug release from patches containing DBP followed Higuchi release model, indicating diffusion controlled release. On the other hand, patches containing DBS followed first order release, indicating that drug release rate was dependent on drug concentration. Patches containing DBS released drug faster than those containing DBP due to the greater uniformity of drug distribution in the polymer matrix and hence higher surface diffusion, and also to the fact that DBS relaxes the chains of the polymer more effectively than DBP, thus increasing the rate of drug diffusion. It has been reported that drug release from a transdermal drug delivery system mainly involves diffusion [18].

It has been reported that linseed oil enhances the permeation of drug by disrupting the lipid structure of stratum corneum, thereby increaseing the diffusion coefficient of the drug across the skin.[19] In the present study, linseed oil is superior to L-menthol in enhancing drug permeation due probably to higher solubility in the polymer matrix, Both the in vitro drug release and skin permeation results indicate skin is a rate-limiting factor in transdermal delivery since the values for the former were higher than those for the latter. The rate of drug permeation was fairly constant over time and the permeation profiles exhibited concentration-dependent firstorder kinetics.

\section{CONCLUSION}

Of all the patches, DB2, which contains $4 \%$ linseed oil, may be is the suitable for transdermal delivery due to its good mechanical and physicochemical properties, uniform drug release/permeation with diffusion-controlled release mechanism. Further studies, including pharmacokinetic investigations, are however required to confirm findings from this study.

\section{ACKNOWLEDGEMENT}

The authors are grateful to the International Medical University (IMU) for funding this study, and to Aurobindo Pharmaceutical Lt., India, for the gift of ondansetron hydrochloride. Thanks also go to the Director of the School of Bioscience and Engineering, Jadavpur University, Kolkata for providing facilities for mechanical studies on the transdermal patches. 


\section{REFERENCES}

1. Rogers MP, Blackburn L. Use of neurokinin-1 receptor antagonists in patients receiving moderately or highly emetogenic chemotherapy. Clinical Journal of Oncology Nursing 2010; 14: 500-504.

2. Bloechl-Daum B, Deuson RR, Mavros $P$, Hansen M, Herrstedt J. Delayed nausea and vomiting continue to reduce patients' quality of life after highly and moderately emetogenic chemotherapy despite antiemetic treatment. Journal of Clinical Oncology 2006; 24(27): 4472-4478.

3. Cohen L, de Moor CA, Eisenberg P, Ming, EE, Hu H. Chemotherapy-induced nausea and vomiting: Incidence and impact on patient quality of life at community oncology settings. Supportive Care in Cancer 2007; 15(5): 497-503.

4. Grunberg SM, Deuson RR, Mavros $P$, Geling $O$, Hansen $M$, Cruciani G, Daniele B, De Pouvourville $G$, Rubenstein $E B$, Daugaard $G$. Incidence of chemotherapy-induced nausea and emesis after modern antiemetics. Cancer 2004; 100(10): 22612268.

5. Ihbe-Heffinger A, Ehlken, B, Bernard, R, Berger, $K$, Peschel C, Eichler HG, Deuson R, Thödtmann1 $J$, Lordick F. The impact of delayed chemotherapyinduced nausea and vomiting on patients, health resource utilization and costs in German cancer centers. Annals of Oncology 2004; 15(3): 526-536.

6. Gwak HS, Oh IK, Chun IK. In vitro Percutaneous absorption of ondansetron hydrochloride from pressure-sensitive adhesive matrices through hairless mouse skin. Arch. Pharm. Res 2003; 26(8): 644-648.

7. Ye JH, Ponnudurai $R$, Schaefer R: Ondansetron. A selective 5-HT3 receptor antagonist and its applications in CNS-related disorders. Neva Press, Branford, Connecticut, 2001; 7(2): 199-213.

8. Gwak HS, Oh IS, Chun IK. Transdermal delivery of ondansetron hydrochloride: effects of vehicles and penetration enhancers. Drug Dev Ind Pharm. 2004; 30(2):187-194.

9. Swain K, Pattnaik S, Sahu SC, Patnaik KK, Mallick S. Drug in adhesive type transdermal matrix systems of ondansetron hydrochloride: optimization of permeation pattern using response surface methodology. J. Drug. Targeting 2010; 18(2): 106114.

10. Hyppola R, Husson I, Sundholm F. Evaluation of physical properties of plasticized ethyl cellulose films cast from ethanol solution Part I. Int. J. Pharm. 1996; 133: 161-170.

11. Rajan R, Sheba NDavid, Khanam J, Nanda A. Studies on the effect of plasticizer on in vitro release and ex vivo permeation from Eudragit E 100 based chlorpheniramine maleate matrix type transdermal delivery system. J. Excipients and Food Chem. 2010; 1(2): 3-11.

12. Li, Ren $C$, Wang $M$, Zhao $L$, Wang $X$, Fang $L$. Optimized preparation and evaluation of indomethacin transdermal patch. Asian. J. Pharm. Sc. $2007 ; 2$ (6): 249-259.

13. Guo JH. An investigation into the formation of plasticizer channels in plasticized polymer films. Drug Dev. Ind. Pharm. 1994; 20(11): 1883-1893.

14. Manning SC, Moore RB. Reactive Compatibilization of Polypropylene and Polyamide- 6, 6 with Carboxylated and Maleated Polypropylene. Polymer Engineering and Science 1999; 39(10): 1921-1926.

15. Repka MA, James WM. Influence of Chlorpheniramine Maleate on Topical Hydroxypropylcellulose Films Produced by Hot-Melt Extrusion. Pharmaceutical Development and Technology 2001; 6(3): 297-304.

16. Gere JM, Timoshenko SP. Mechanics of Materials, 3rd edn, PWS-KENT Publishing Company, Boston, MA, 1990

17. Siepmann J, Lecomte $F$, Bodmeier $R$. Diffusion controlled drug delivery systems: calculation of the required composition to achieve desired release profiles. Journal of Controlled Release 1999; 60(23): 379-389.

18. Sogol K, Ismaeil $H$, Yaghob Physico-mechanical analysis of free ethyl cellulose films comprised with novel plasticizers of vitamin resources. Int Journal of Pharmaceutics 2008; 356: 153-166.

19. Inderbir S, Pradeep K, Harinderjit S, Malvika G, Vikas $R$. Formulation and evaluation of domperidone loaded mineral oil entrapped emulsion gel (MOEG) buoyant beads. Acta Pol Pharm. 2011; 68(1): 121 126. 